\title{
Foreword
}

\section{Molecular Signatures of Infectious Agents in Human Cancer}

\author{
Mukesh Verma* and Sudhir Srivastava \\ Cancer Biomarkers Research Group, Division of \\ Cancer Prevention, National Cancer Institute, NIH, \\ Room 330E, Executive Plaza North, 6130 Executive \\ Boulevard, Rockville, MD 20852-7346, USA
}

As of 2000, experimental and epidemiological evidence has firmly established an association between various cancer sites and at least eight viruses, a few parasites (Schistosomes and liver fluke), and one bacterium (Helicobacter pylori). Approximately $15 \%$ of cancers worldwide, and $23 \%$ of cancers in developing countries, are now attributed to infectious agents. Most of this burden is related to viral infections (i.e, $10 \%$ worldwide, $17 \%$ in developing countries). Infectious agents represent, therefore, priorities in cancer prevention research.

Cancer-associated viruses likely act, at least in part, by predisposing the cell to genetic changes, resulting in the progressive steps that lead to cancer growth. It may be beneficial, therefore, to identify early mutational changes in the progression to cancer. While involving primarily a distinct set of technologies from that used in developing molecular signatures, the knowledge gained from generating a genetic profile of cancer and its precancerous lesions may help predict the molecular signature of these lesions.

Improvements in the detection of viruses and biomarkers of chronic infection have led to the identification of strong associations with cancer, particularly for human papillomavirus (HPV), hepatitis B virus (HBV), and human immunodeficiency virus (HIV). For some cancer associated viruses (e.g., HIV and hepatitis

*Address for correspondence: Mukesh Verma, Ph.D., Program Director, Cancer Biomarkers Research Group, Division of Cancer Prevention, National Cancer Institute, National Institutes of Health, Room 3144, Executive Plaza North, 6130 Executive Boulevard, Rockville, MD 20852-7346, USA. Tel.: +1 301496 3893; Fax: +1 301402 8990; E-mail: mv66j@nih.gov.
$\mathrm{C}$ virus, $\mathrm{HCV}$ ), the spectrum of malignancies involved has still to be well defined. These improvements, in part, have been facilitated by our ability to measure gene expression.

Gene expression profiling provides a powerful means for defining molecular signatures for diseases. Molecular signatures permit one to identify patterns of changes both at the RNA (gene chips/arrays) and protein (proteomics) levels between any given stage of cancer. They allow one to screen effectively for changes regardless of the level of knowledge that exists regarding the viral mechanisms of action in cancer. The success of this approach is based upon the availability and quality of the samples being compared. Human samples are optimal, where available; however, for many human cancers, laboratory animal models or tissue culture models may be more practical sources of reproducible materials for analysis.

While gene expression profiling approaches may be beneficial, the availability of matched tissue samples or validated animal models may limit their application. For many viruses, there is arising, through traditional reductionist scientific investigative efforts, fundamental insights into the mechanism by which they contribute to cancers. From these insights arise predictions for molecular signatures for virally-induced cancers. Thus, research initiatives that test the validity of these predicted molecular signatures may provide great potential, especially where gene expression profiling studies are not feasible or as an augmentative approach to the latter. In all known cases, viruses that are associated with cancers are ones that persist for long periods in the host. How the host responds to the virus and how the virus modulates this response must be critical in allowing for viral persistence and indeed may be determinative of the risk of cancer. Both the general immune competence and the type of immune response (e.g. type I versus type II) may be determinative of the outcome of the viral infection and subsequent risk of cancer development. In some cases, as with HBV 
and perhaps also HCV, cancer may result from an underlying immunopathological disease. Specific haplotypes may confer risk for and protection from cancer; for instance, controversy surrounds assertions that haplotypes in class-II genes and extended haplotypes that include class-I-linked genes help determine risk of cervical cancer. In addition, oncogenic viruses, in many cases, have learned to evade the immune system; such evasion strategies likely contribute to their oncogenic potential. Therefore, the immunobiology of persistent viral infections, and in particular those that lead to cancer, must be better understood. This knowledge could contribute not only in helping identify patients at risk of developing cancer, but also in developing immunological intervention strategies.

Validated laboratory animal models for virallyinduced human cancers will be pivotal to the success of a molecular signatures program. For some cancers, laboratory animal models may be essential for providing the critical tissue samples with which to identify molecular signatures, not only for the frank cancer but more importantly, the precancerous lesions that for some human cancers are difficult to identify or obtain. Animal models will also provide the means for testing the validity of a molecular signature, understanding the underlying mechanism of action by which the viral agent contributes to the molecular signature, and providing a means of testing new therapeutic modalities for intervening in virally induced cancers that might arise as a consequence of defining molecular signatures. Thus, a molecular signatures program will need to encompass the development and use of validated animal models for virally-induced human cancers.

The Cancer Biomarkers Research Group (CBRG) of the Division of Cancer Prevention (DCP), National Cancer Institute, sponsored a workshop entitled, "Molecular Signatures of Infectious Agents" in Bethesda, Maryland, September 7-8, 2000 to identify molecular signatures of infectious agents and to utilize this information for risk assessment and development of prevention strategies against these infectious agents. The specific objectives of the workshop were to review state-of-the-science in detection technology that can identify extraneous genomic insertion in human cancers and to establish future research directions for using the molecular signatures of infectious agents for early detection, risk assessment, and prevention of cancer. This meeting attracted 70 scientists actively engaged in research on infectious agents and human cancer. Sessions included an overview of infectious agents and their interaction with the host, contribution of infectious agents in the development of disease, technology advancement in detecting multiple infections, role of growth factors and cytokines in cancer progression, animal models in understanding host-virus interaction, and development of prevention strategies against infectious agents. In this special issue of Disease Markers, some of the participants have discussed their research in the intriguing field of cancer and infectious agents.

Strategies to reduce the risk of virus-related cancers include: avoidance of infection by screening for cancer viruses in traditional sources (e.g., blood and blood derivatives, cervical smears) or carriers (e.g., pregnant women, organ donors, etc.), active host immunization through prophylactic and therapeutic vaccines, and antiviral treatment to prevent chronic carrier status. For HBV and HPV, vaccination aimed at cancer prevention is already a reality. Whereas HBV vaccination already emerged as one of the most cost-effective ways to reduce adult cancer mortality, HPV vaccination still awaits solution for technical problems. For other infectious agents, prospects for a vaccine are not immediate.

In order to apply new knowledge about viruses to cancer prevention, large vaccination trials are warranted. These will have to be large (many thousands of people), prolonged (5-10 years), and match scientific excellence with a feasible design. 


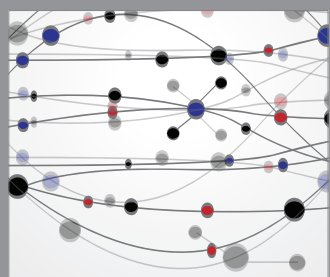

The Scientific World Journal
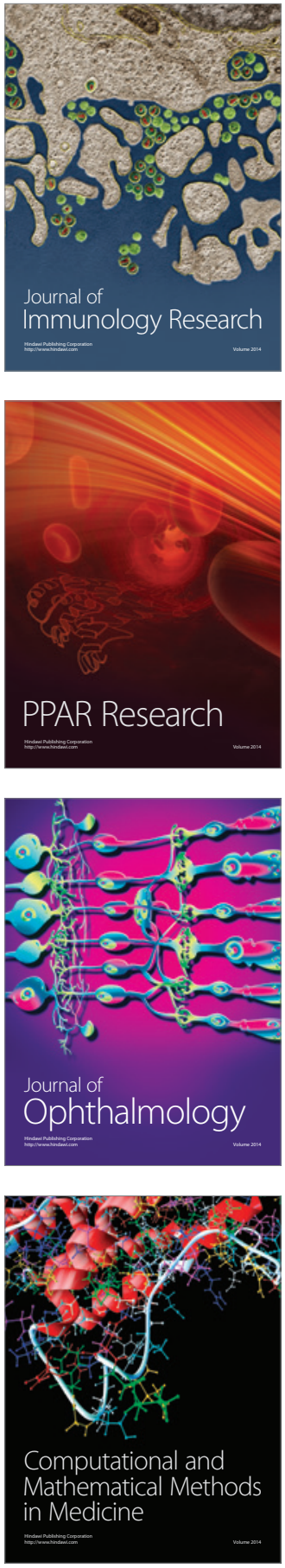

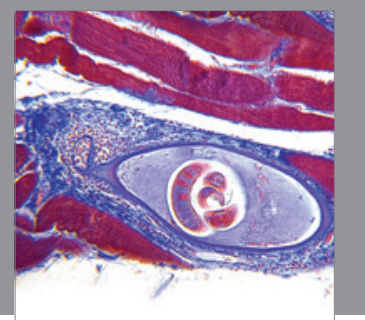

Gastroenterology

Research and Practice
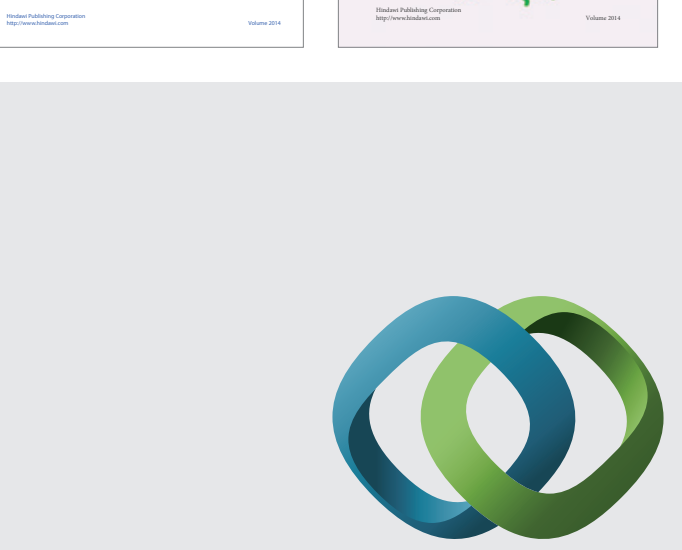

\section{Hindawi}

Submit your manuscripts at

http://www.hindawi.com
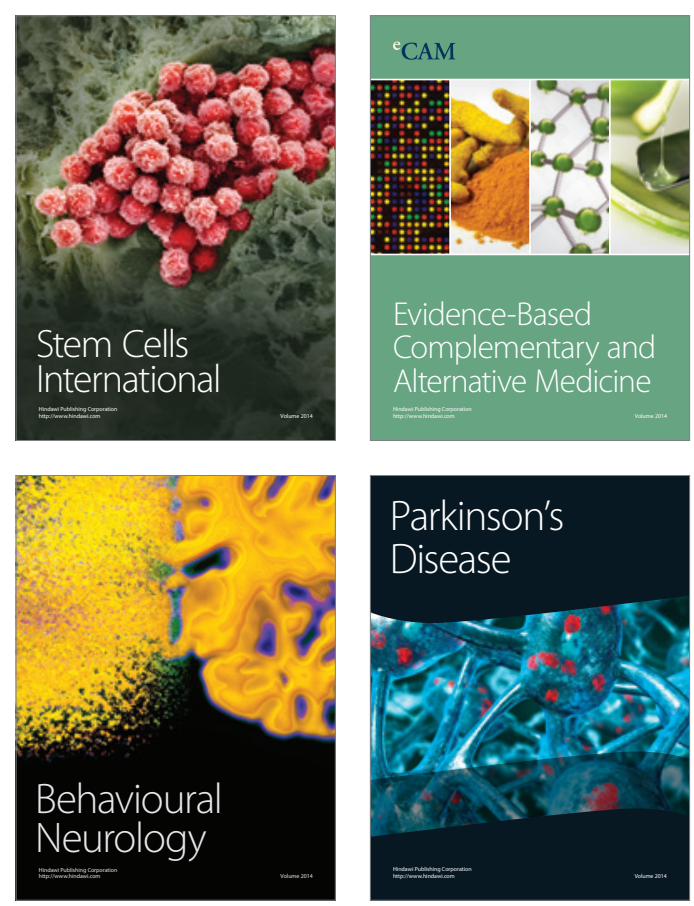

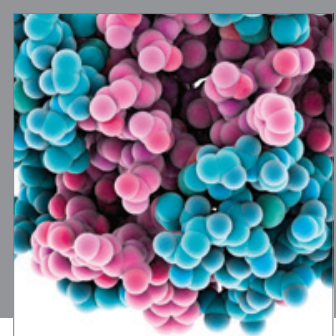

Journal of
Diabetes Research

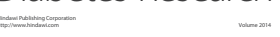

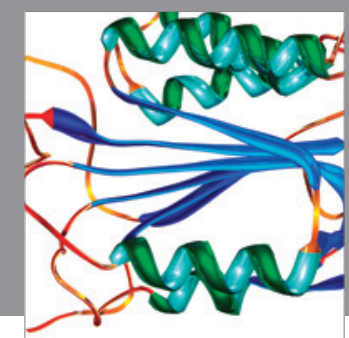

Disease Markers
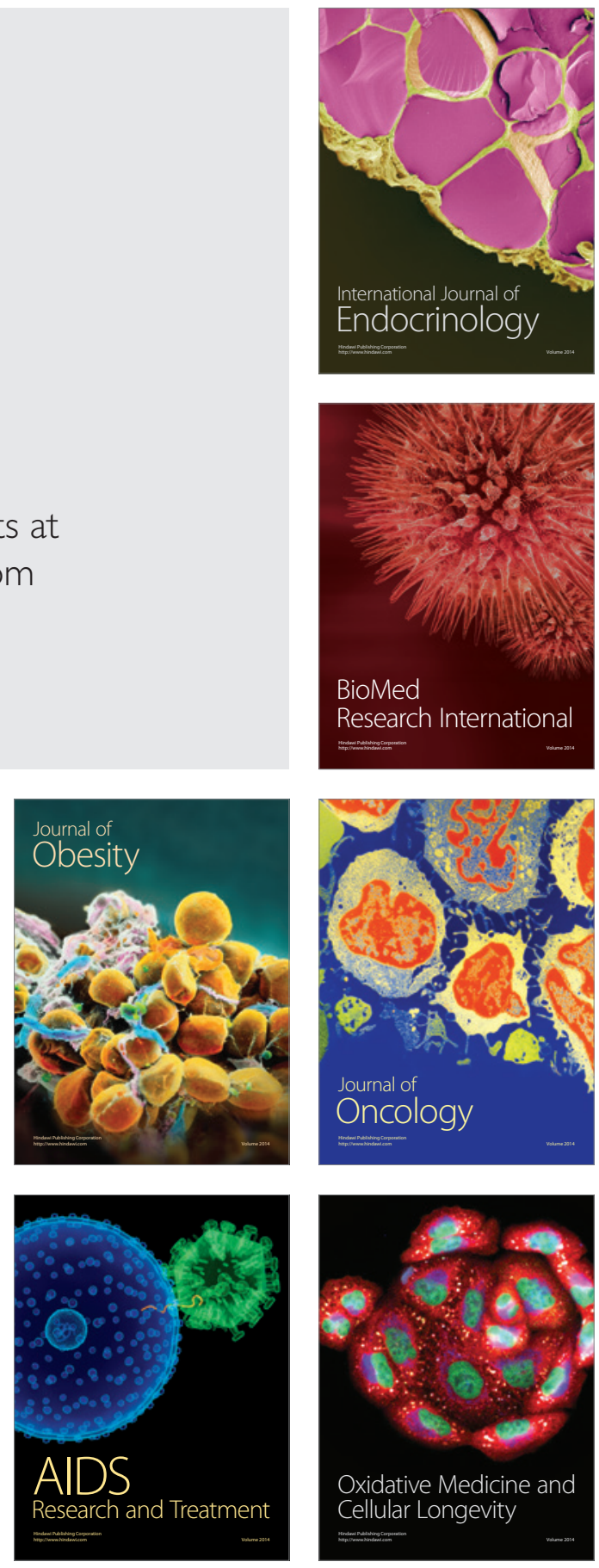